\title{
Man-made Object Detection Based on Texture Clustering and Geometric Structure Feature Extracting
}

\author{
Fei Cai \\ Key Laboratory for Information System Engineering, School of Information System and Management, \\ National University of Defense Technology, Changsha, China \\ E-mail: fei525lin@163.com \\ Honghui Chen, Jianwei Ma \\ Key Laboratory for Information System Engineering, School of Information System and Management, \\ National University of Defense Technology, Changsha, China \\ E-mail: \{chh0808, majianwei.nudt\}@google.com
}

\begin{abstract}
Automatic aerial image interpretation is one of new rising high-tech application fields, and it's proverbially applied in the military domain. Based on human visual attention mechanism and texture visual perception, this paper proposes a new approach for man-made object detection and marking by extracting texture and geometry structure features. After clustering the texture feature to realize effective image segmentation, geometry structure feature is obtained to achieve final detection and marking. Thus a man-made object detection methodology is designed, by which typical man-made objects in complex natural background, including airplanes, tanks and vehicles can be detected. The experiments sustain that the proposed method is effective and rational.
\end{abstract}

Index Terms-man-made object detection, image segmentation, object marking, feature extraction, texture clustering

\section{INTRODUCTION}

As a key technology of image processing, man-made object detection is the basis for target tracking and recognition, widely applied in intelligent transportation [1, 2], urban planning [3, 4], damage assessment [5-7] and military target detection [8]. Automatic detection and marking of man-made object will improve the efficiency of remote sensing image interpretation, and be beneficial to the flight control of unmanned aerial vehicles (UAV). It has great significance for intelligence obtaining and situation grasping of a war.

Several man-made object detection methods, based on geometric structure, fractal feature, probability models, level set and clustering, are generalized in current research [9]. Many approaches published recently rely on priori information. Traditional algorithms are based on edge matching between an observation, the image and a prediction [10]. Some others processed various shape characteristics, like Zernique moments, on target segmentation [11]. Reference [12] describes a man-made object segmentation method for aerial images based on a modified watershed segmentation algorithm by three steps, namely a multi-scaled geometric image analysis, watershed segmentation and region classification of man-made objects. Its experimental results show that the outcome of man-made objects segmentation becomes more continuous and satisfying. Jun Yang [13] proposed an attention driven level set method for extracting manmade regions from aerial or satellite images. Compared with other level-set segmentation, the main remodification of the novel approach artfully avoids much redundant computation and pops out the efficiency perceptually. Based on the assumption that targets and backgrounds have different textures, in [14], Xavier et al. used a boosting algorithm to select discriminating features and introduced a new descriptor--Histogram Distance on Haar Regions (HDHR), robust to background and target texture variations, to realize automatic object detection on aerial images Observed results prove that it can be trained on adapted simulated data and yet be efficient on real images, compared with several classical descriptors.

In recent years Kernel Principal Component Analysis (Kernel PCA) has been successfully employed to capture nonlinear image features to achieve remarkable object detection by Saad Ali and Mubarak Shah [15]. They presented a novel framework for object detection that combines feature reduction and selection, having been successfully tested on wide range of objects (cars, airplanes, pedestrians, motorcycles, etc) in standard data sets and shown advisable performance. Followed by previous researches with regard to target detection based on visual attention [16, 17], Hae Jong Seo [18] and Wei $\mathrm{Li}$ [19] presented respective visual saliency-based automatic object detection methods. Unalterably, their experimental results informed that their work provides a promising way to solve related problems.

Generalizing from related researches, the main difficulty lies in obtaining significant distinctions between man-made objects and natural background to achieve effective image segmentation, resulting in high false detection. Based on human visual perception, a novel man-made object detection approach referred to 
texture feature clustering and geometric structure feature extracting is proposed, which chooses suitable features including contrast, edge, and corner. Theoretical analysis and experimental comparison verifies the effectiveness and rationality of proposed approach. This paper is organized as follows. Section I briefly surveys related researches and applications while Section II gives the background of the problem. The main idea of proposed approach is described in Section III while experimental results and discussions are elucidated in Section IV. Section $\mathrm{V}$ gives the conclusions and future directions of research.

\section{BACKGROUND}

In image processing field, man-made object detection can be classified into image segmentation with specific restrictions. A two-dimensional aerial image $G(x, y)$ can be described as (1).

$$
G(x, y)=B(x, y)+\sum_{i=1}^{N} O_{i}(x, y)+N(x, y)
$$

where $B(x, y)$ represents natural background, $O_{j}(x, y)$ means man-made objects and $N(x, y)$ denotes noises. Then man-made object detection diagram can be briefly described as Fig. 1. Combining with practical application, this paper proposes following three assumptions: (1) Visual discontinuity between objects and background does exist. That is the emergences of objects are seen as abnormities of the whole scene; (2) The geometric structure features of man-made objects, including line edges or obvious corners and so on, are more prominent than background; (3) The frequency of occurrence of man-made objects is lower compared to the background, in other words, the total number of background pixels in an image is larger than objects.

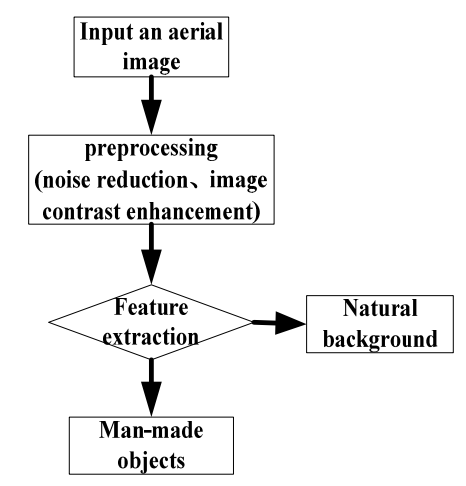

Figure 1. Man- made object detection diagram.

\section{PROPOSED ALGORITHM}

This paper proposes a new algorithm for man-made object detection by clustering the texture feature available to human visual system and extracting the geometric structure features. This algorithm includes three modules: preprocessing module, primary location module and final marking module. The general experimental framework is shown in Fig. 2. The left part of Fig. 2 is the chain process while the right part explicates each process module.

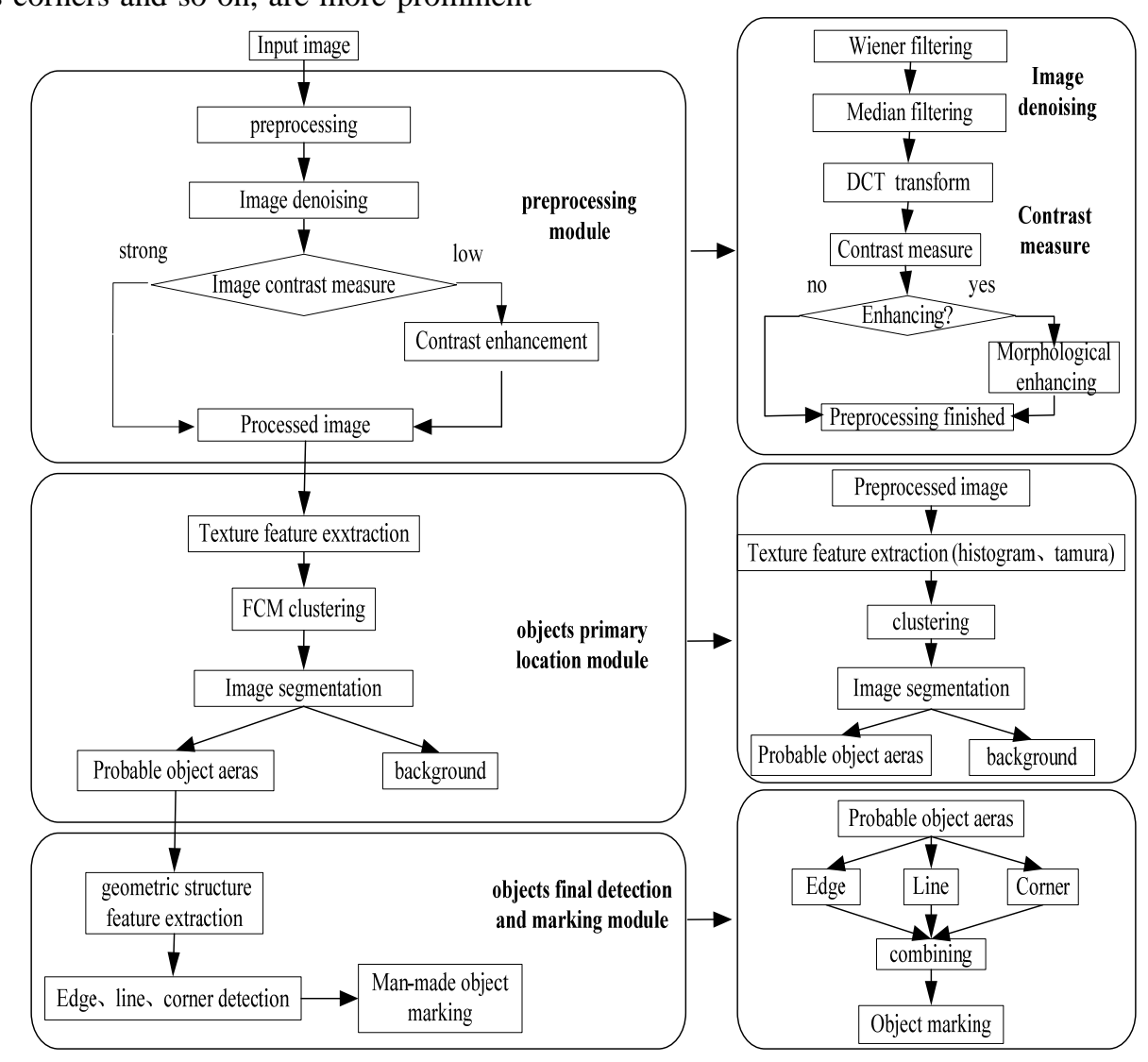

Figure 2. Experimental framework. 


\section{A. Preprocessing}

Salt-and pepper noise in acquiring aerial images with a CCD camera and Gaussian noise caused principally by image transmission are taken into account. Furthermore, we enhance the contrast of image by mathematic morphology after measuring it based on DCT coefficients. It is known that object detection by human visual system depends on the ratio between highfrequency and low-frequency content [20]. Thus, the contrast can be measured as the ratio of high-frequency and low-frequency content in the bands of the DCT matrix [20, 21]. Let $\left\{d_{k, l}\right\}$ be the DCT transform of $8 \times 8$ block in the original image. We first classify the coefficients into 15 different frequency bands. The $n$th band is composed of the coefficients with $n=k+l(0 \leq k, l \leq 7)$. The measurement at the $n$th band is defined as (2).

$$
C_{n}=\frac{E_{n}}{\sum_{t=0}^{n-1} E_{t}}
$$

where

$$
\begin{gathered}
E_{t}=\frac{\sum_{k+l=t}\left|d_{k, l}\right|}{N} \\
N= \begin{cases}t+1, & t<8 \\
14-t+1, & t \geq 8\end{cases}
\end{gathered}
$$

Table I shows the comparison of images with the same scene but distinct contrast. We conclude that images with strong contrast usually have larger values $c_{i}$, and from repeated experiments, 0.2 is chosen to be the threshed to determine whether the contrast of image is strong or not.

\begin{tabular}{|c|c|c|c|}
\hline Contrast & Images & C1 & $\mathrm{C} 2$ \\
\hline Low & & 0.1874 & 0.0965 \\
\hline Strong & & 0.2131 & 0.1098 \\
\hline
\end{tabular}

TABLE I.

COMPARISON OF IMAGES WITH DISTINCT CONTRAST

\section{B. objects primary location}

This paper verifies different primary location approaches based on gray value and texture feature respectively. For choosing ideal texture feature, the experiments compare the clustering results of different statistics between histogram-based and Tamura-based texture feature. Ultimately, contrast based on Tamura is adopted and FCM is employed as the clustering algorithm for its low computational consumption and effective segmentation.

One simple approach for describing texture is to use statistical moments of histogram. Let $Z$ be a random variable denoting gray levels. The $n$th moment of $Z$ about the mean is described as (5).

$$
\mu_{n}=\sum_{i=0}^{L-1}\left(z_{i}-m\right)^{n} p\left(z_{i}\right)
$$

where $L$ is the number of gray levels, and $m$ is the mean value of $Z$.

$$
m=\sum_{i=0}^{L-1} z_{i} p\left(z_{i}\right)
$$

Statistics $m$ measures the brightness while standard deviation $\sigma$ measures the contrast.

$$
\sigma=\sqrt{\mu_{2}(z)}=\sqrt{\sigma^{2}}
$$

Tamura [22] proposed other statistics to describe texture feature based on the psychological research on visual perception, including coarseness, contrast and so on. The contrast also can be derived as (8).

$$
F_{\text {contrastness }}=\frac{\sigma}{\alpha_{4}^{1 / 4}}
$$

where $\alpha_{4}=\mu_{4} / \sigma^{4}, \mu_{4}$ is the 4 th moment and $\sigma^{2}$ is the variance. Fig. 3 shows the contrast distinction between man-made object areas and background. In Fig. 3(b), Xaxis indicates the horizontal location of a window in an image while $\mathrm{Y}$-axis reflects the $F_{\text {contrastness }}$ value of a removable window with the same vertical coordinate. There is a sharp peak in areas containing man-made objects while the curve is plain in other background areas. This characteristic can be used to distinguish the property of the window.

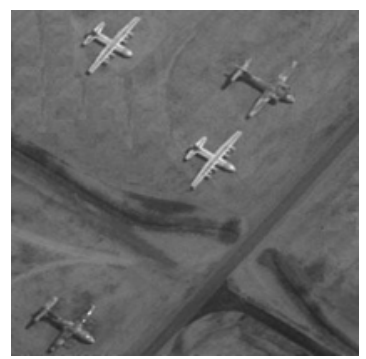

(a) Original image

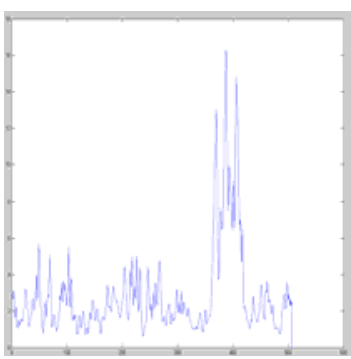

(b) contrast feature
Figure 3. Comparison of contrast

\section{C. objects final detection and marking}

For geometric structure features of man-made objects are obvious based on assumption 2, man-made objects can be marked by additional line edges and corners detection after primary location by clustering the texture feature. Susan operator [23] is employed to detect the 
corners and the edges are detected by Tupin approach $[24,25]$, then lines are extracted by Hough line detection. Fig. 4 shows the partial experimental results of edge detection. We can see that edges detected by Tupin accord better with the real edges of objects than Canny.

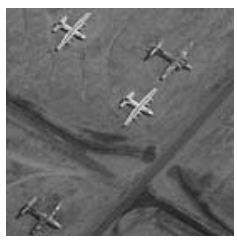

(a) Original image

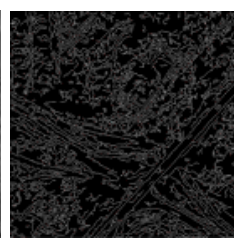

(b) Canny

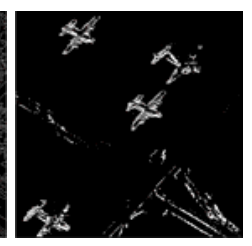

(c) Tupin approach
Figure 4. Distinct edge detection results

\section{PROPOSED ALGORITHM}

As shown in figure 2, the experiment is carried out as follows:
(1) Input a 2-D gray image, measure its contrast and enhance it when needed followed by denoising with Weiner and median filter in turn,

(2) Choose an appropriate window, extract different texture feature of the window;

(3) Cluster the texture feature to realize image segmentation compared to gray value by FCM clustering algorithm,

(4) Extract the geometric structure feature, including edges, lines, and corners, to mark the objects.

Our experiment is implemented on aerial images from USC-SIPI image database, and the software environment is Matlab7.8.0. We adopt a $3 \times 3$ window when denoising image while a disk-shaped structuring element with 3 as its radius is used to enhance the contrast. Table II shows results of preprocessing.

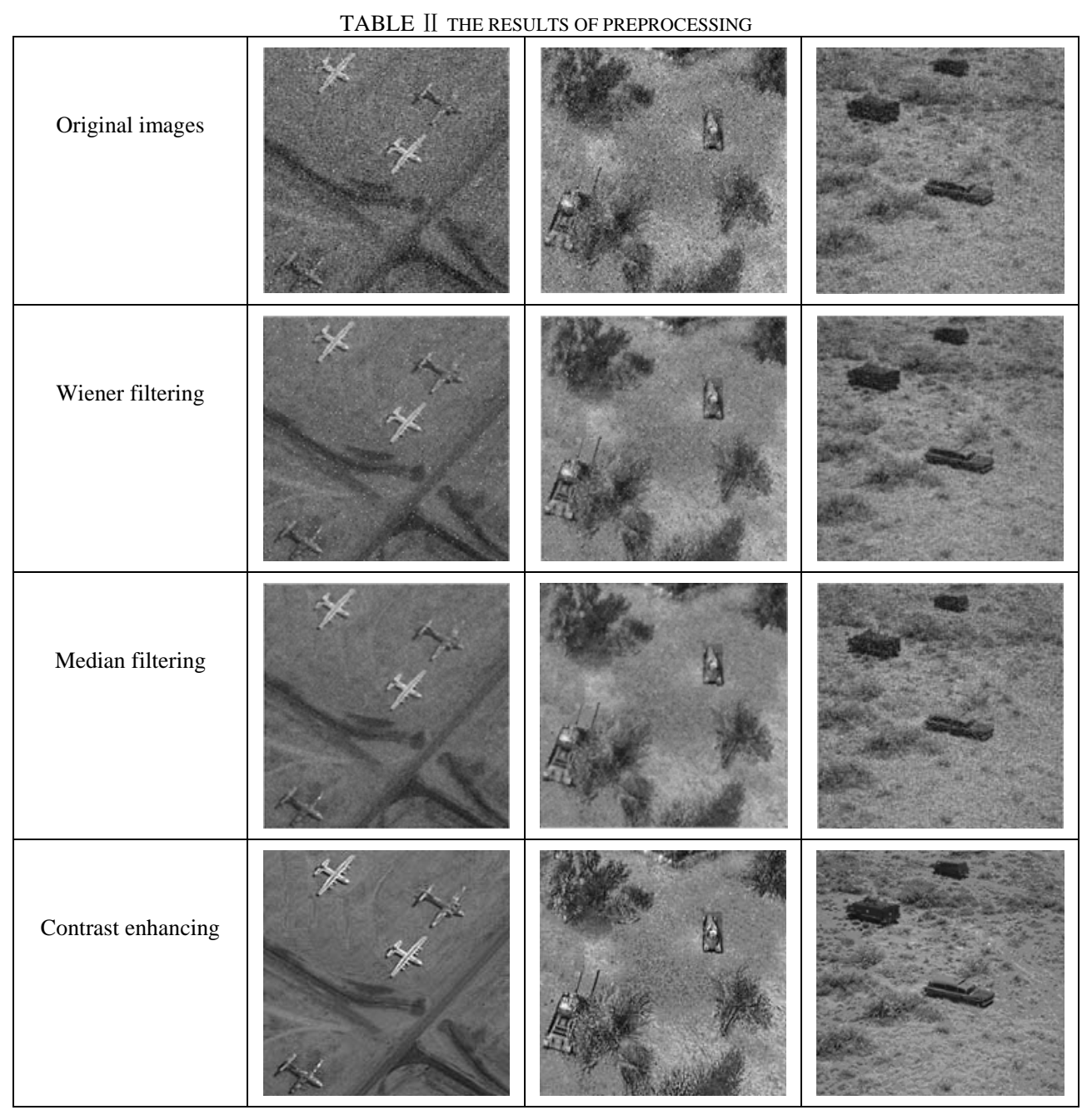

In a $5 \times 5$ window, texture features are computed as the value of central pixel. Image segmentation is accomplished by FCM. Table III presents the clustering results and comparison with [26]. That the number of clusters is 2 means an image is clustered into 2 portions, namely background and object areas. Euclidean distance and Mahalanobis distance are adopted when clustering one feature statistic and feature vector, respectively. Comparing row 5 in Table III with other rows, results based on clustering to Tamura texture feature contrast are unambiguously more accurate besides lower complexity. 


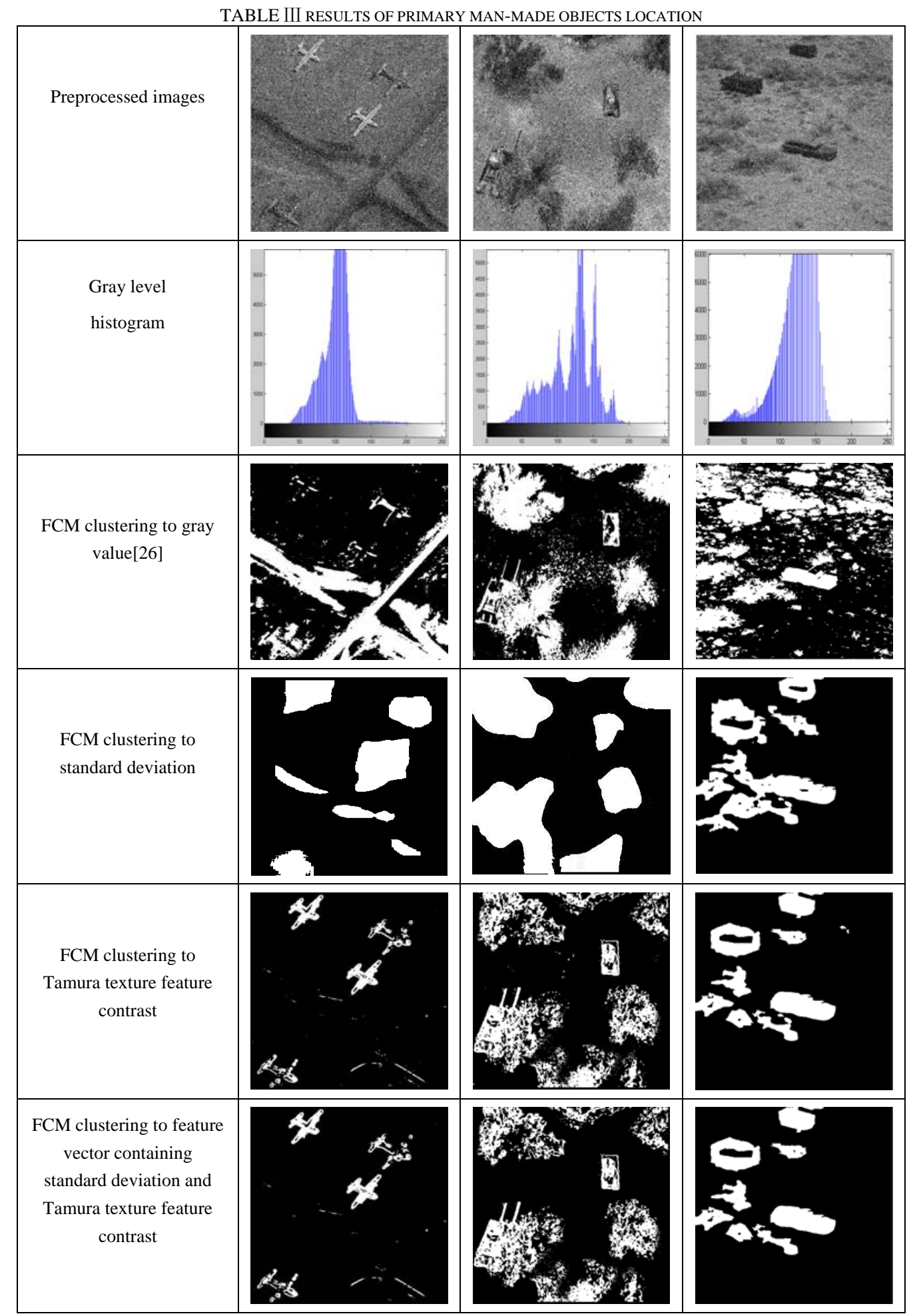

Table IV shows the results of man-made objects marking. Red areas denote detected objects. Some longer lines and sharper corners are preserved due to assumption 2. The detected red lines approximate to the real object's edges, accompanied with some false lines. Furthermore, there's few distinction between our method and artificial man-made objects marking. This may be caused by errors when extracting geometric structure features, or the following marking rules: in primary object location areas, choose a window containing both line edges and enough corners as object candidates, get a pixel by computing the average of coordinate of corners as the center of a $5 \times 5$ square to mark the objects. 
TABLE IV MAN-MADE OBJECTS MARKING (RED)

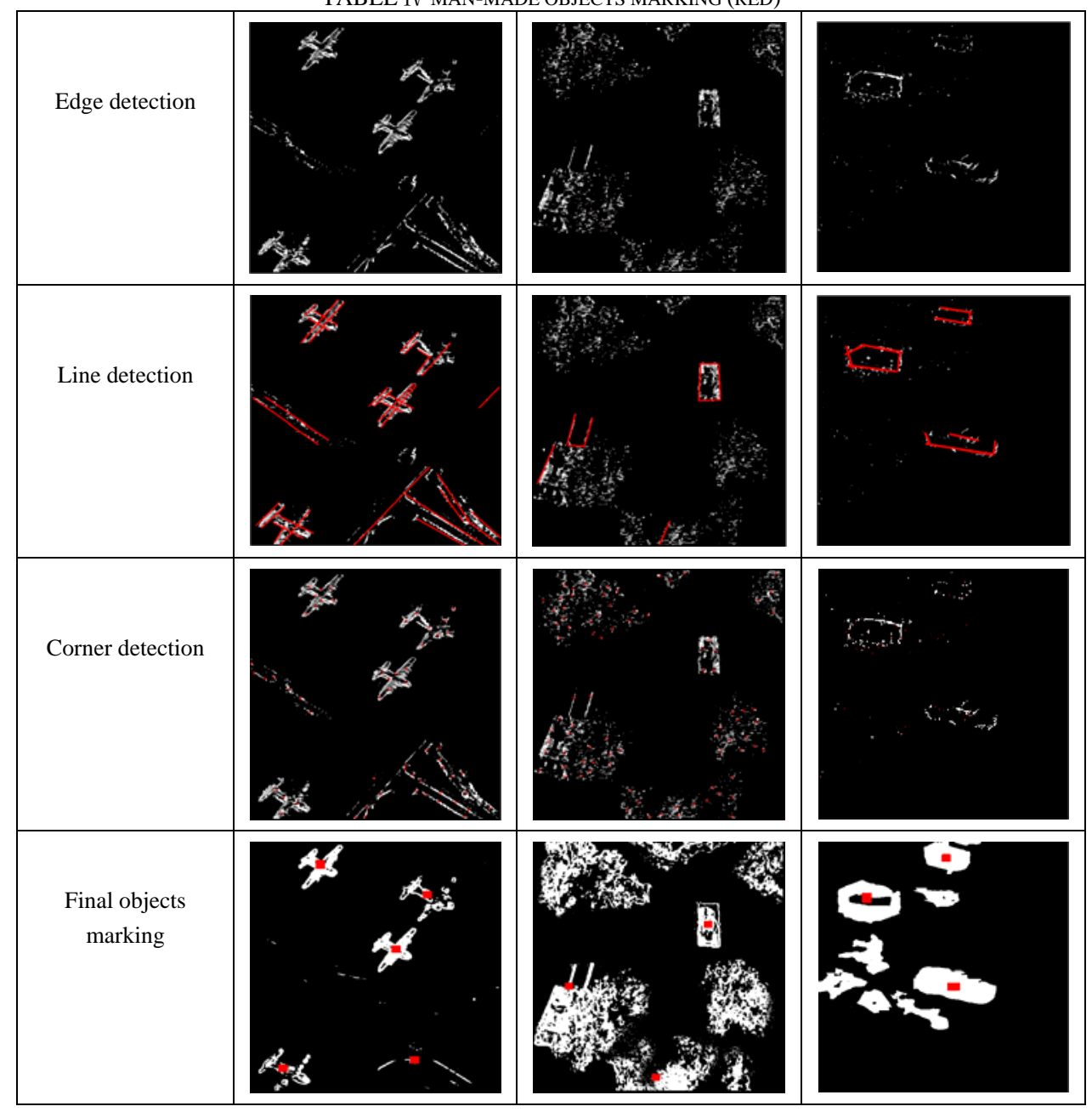

Table $\mathrm{V}$ shows the advantages and defects of proposed approach based on ATR evaluation index. The computational complexity mainly depends on texture feature extraction. We conclude that the false alarm probability of proposed approach is usually high in respect that the chosen features of natural background are analogical with man-made objects. Consequently, this provides a guider for our further research. In order to verify the feasibility of our method, other aerial images from USC-SIPI database are tested. Table VI shows additional experiment results. Man-made objects from clutter background are veraciously detected.

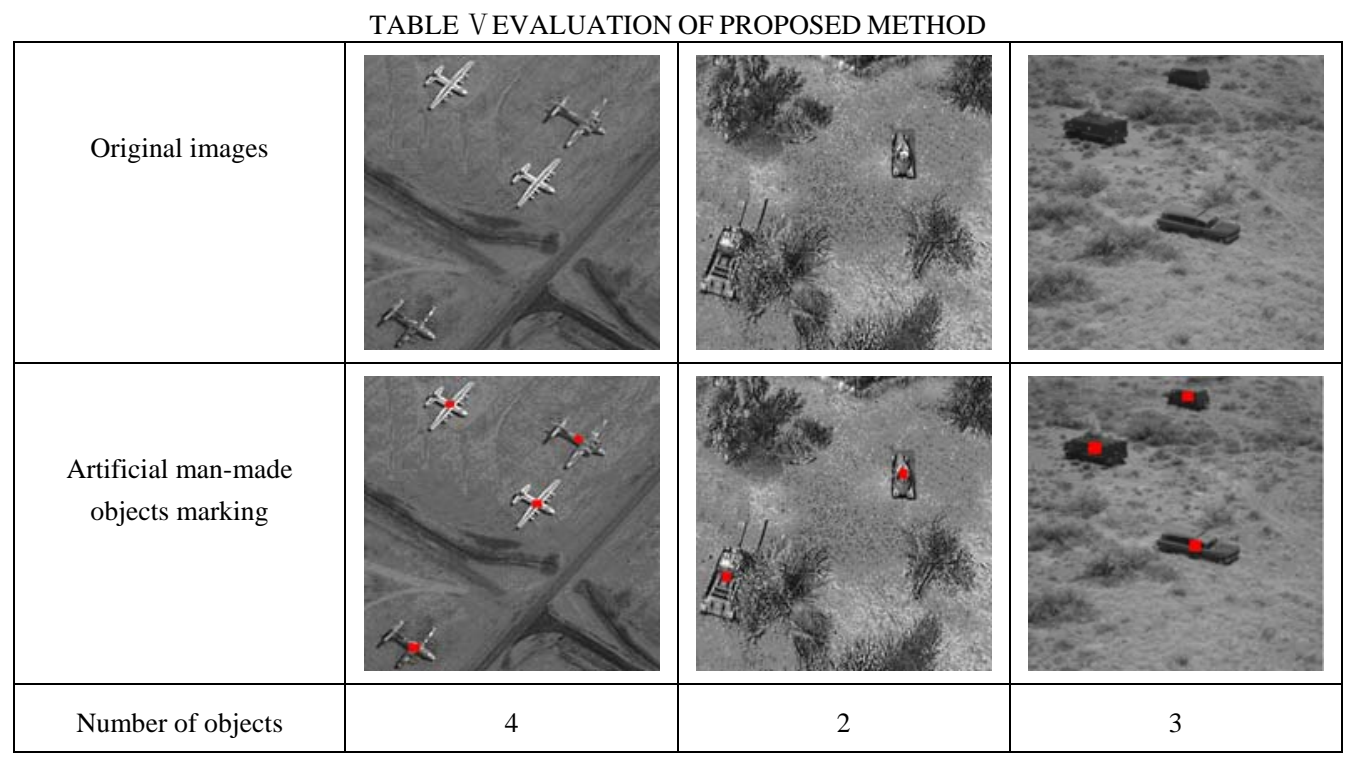




\begin{tabular}{|c|c|c|c|}
\hline Automatic detected & $\mid 2$ & \\
\hline Automatic marking & 5 & excellent \\
\hline Detection precision & excellent & 0 & 0 \\
\hline False alarm probability & $25 \%$ & 0 & 0 \\
\hline $\begin{array}{c}\text { Probability of missed } \\
\text { detection }\end{array}$ & 0 & 0 & 0 \\
\hline
\end{tabular}

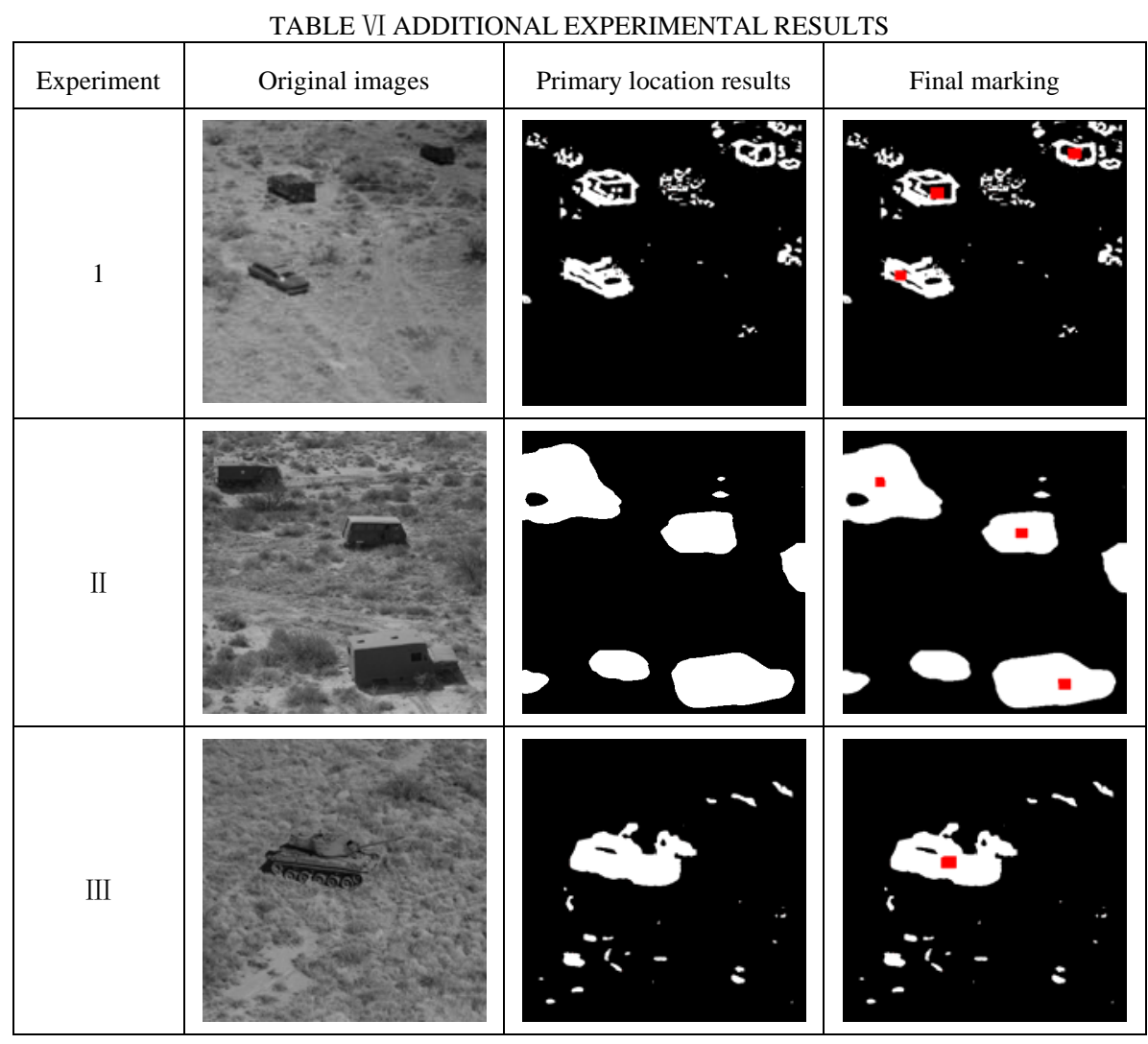

\section{CONCLUSION}

This paper proposes an automatic man-made object detection methodology involving image denoising, contrast enhancing, feature extraction, image segmentation and object marking. Man-made objects can be detected based on clustering reliable texture feature and extracting geometric structure features. The experiments on distinct aerial images containing typical objects verify the rationality and validity of proposed approach.

From current researches, we may conduct further research on following facets: (1) Feature choosing and extracting. Powerful features provide impactful support for man-made object detection. (2) Man-made object classification and recognition. It has great significance for more detailed intelligence obtaining. Incorporating prior knowledge, as vehicles associate with rectangular shapes while airports correlate with two parallel lines, objects can be tagged with semantic understanding and description, which pave the way for object recognition. (3)We will attempt to choose a reliable window when doing preprocessing and feature extracting by theoretical detrusion from current researches.

\section{ACKNOWLEDGMENT}

Thanks to USC-SIPI image database providing the test aerial images, and thanks to Professor M. J. Carlotto from Carnegie-Mellon University for his careful suggestion of our experiment. His comments and 
suggestion have contributed greatly to the depth and clarity of the presentation.

\section{REFERENCES}

[1] Li Bo, Qi Mei Chen, Guo Fan. Freeway Auto-surveillance From Traffic Video[C]. 2006 6th International Conference on ITS Telecommunications Proceedings. 2006. 167-170.

[2] S. Alvarez, M.A.Sotelo, M. Ocana et al. Perception Advances in Outdoor Vehicle Detection for Automatic Cruise Control[J]. Robotica. 2010, 28(5):765-779.

[3] Hinz S, Baumgartner A. Automatic Extraction of Urban Road Networks from Multi-view Aerial Imagery[J]. ISPRS Journal of Photogrammetry \& Remote Sensing, 2003, 58:83-98.

[4] Mourad Bouziani, Kalifa Goita, Dongchen He. Automatic Change Detection of Buildings in Urban Environment from Very High Spatial Resolution Images Using Existing Geodatabase and Prior Knowledge[J]. ISPRS Journal of Photogrammetry and Remote Sensing. 2010,65:143:153.

[5] Turker,M. Sumer.E. Buildings-based Damage Detection Due to Tarthquake Using the Watershed Segmentation of the Post-event Aerial Images[J]. International Journal of Remote Sensing. 2008, 29(11).3073-3089.

[6] Li Peijun, Xu Haiqing, Guo Jiancong. Urban Building Damage Detection from Very High Resolution Imagery Using OCSVM and Spatial Features[J]. International Journal of Remote Sensing, 2010, 31(13): 3393-3409

[7] Supannee Tanathong, Kurt T.Rudahl, Sally E.Goldin. Object Oriented Change Detection of Buildings after the Indian Ocean Tsunami Disaster [C].Proceedings of ECTICON. 2008(1): 65-68.

[8] Jianxin Mei, Duan Shan, Qianqing Qin. Method for Special Targets Detection Based on Support Vector Machines[J]. Geomatics and Information Science of Wuhan University 2004.29(10): 912-915.

[9] Cai Fei, Tu Dan. Survey on Man-made Object Detection in Visible Imagery[J]. Application Research of Computers, 2010, 27(7): 2430-2434.

[10] Ruch, O, Dufour, J. Real-time. Automatic Target Recognition and Identification of Ground Vehicles for Airborne Optronic Systems[J]. Proceedings of the SPIE, 2005(5909): 11-20.

[11] Chen JianMing, Han ChinChuan, Fan KaoChin. Aircraft Type Recognition in Satellite Images[J]. IEEE Proceedings: Vision, Image and Signal Processing. 2005, 152(3):307-315.

[12] Wang Wei, Yang Xin. Rapid Man-made Object Morphological Segmentation for Aerial Images Using A Multi-scaled Geometric Image Analysis[J]. Image and Vision Computing 2010(28):626-633.

[13] Jun Yang, Peng Zhang, Runsheng Wang. Extracting Manmade Region(s) Based on Attention Driven Level-set Evolution[C]. Fourth International Conference on Image and Graphics. 2007(117):465-470.

[14] Xavier Perrotton, Marc Sturzel, Michel Roux. Automatic Object Detection on Aerial Images Using Local Descriptors and Image Synthesis[C].ICVS 2008, 2008(5008): 302-311.
[15] Saad Ali, Mubarak Shah. A Supervised Learning Framework for Generic Object Detection in Images[C]. Proceedings of the Tenth IEEE International Conference on Computer Vision, 2005(8):1347-1354.

[16] Laurent Itti, Christof Koch. A Saliency-based Search Mechanism for Overt and Covert Shifts of Visual Attention [J].Vision Research 2000(40):1489-1506.

[17] Itti, L. Visual Attention and Target Detection in Cluttered Natural Scenes[J]. Optical Engineering, 2001, 40(9):17841793.

[18] Hae Jong Seo, Peyman Milanfar. Visual Saliency for Automatic Target Detection, Boundary Detection and Image Quality Assessment[C].ICASSP 2010.5578-5581.

[19] Wei Li, Chunhong Pan, Li-xiong Liu. Saliency-based Automatic Target Detection in forward Looking Infrared Images [C]. ICIP, 2009:957-960.

[20] E.Peli, Contrast in Complex Images[J]. Optical Society of America, 1990,7(10):2032-2040,

[21] Jinshan Tang, Scott Acton. Image Enhancement Using a Contrast Measure in the Compressed Domain[J]. IEEE Signal Processing Letters. 2003, 10(10): 289-292.

[22] Peter Howarth, Smfan M. Ruger. Evaluation of Texture Feature for Content-based Image Retrieval[C]. Third International Conference, CIVR, 2004: 326-334

[23] Smith S M, Brady M .Susan. A New Approach to Low Level Image Processing[J]. International Journal of Computer Vision 1997.23(1):45-78.

[24] Ridha Touzi, Arand Lopes, Pierre Bousquet. A Statistical and Geometrical Edge Detector for SAR Images[J]. IEEE Transaction on Geoscience and Remote Sensing.1988, 26(6):764-773.

[25] Yingying Chen, Zhaohui Yang, Qun Su. Automatic Recognition of Man-made Objects in SAR Images Using Support Vector Machines[J]. 2009 Urban Remote Sensing Joint Event 9(9):78-83.

[26] M. J. Carlotto. A Cluster-based Approach for Detecting Manmade Objects and Changes in Imagery[J], IEEE Trans on Geoscience and Remote Sensing, 2005,43(2):374-387

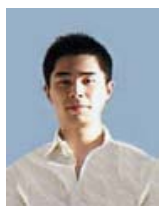

Fei Cai was born in Nantong, Jiangsu Province, China on November 4th 1984. Cai is now a Ph.D. candidate at National University of Defense Technology. Cai was awarded the Degree of Bachelor of Engineering in Biomedical Engineering in Nanjing University of Aeronautics and Astronautics (NUAA), Nanjing, China on July 1st, 2008, and he received his Degree of Master of Engineering in Control Science and Engineering in Multimedia and Virtual Reality in National University of Defense Technology (NUDT), Changsha, China on December 1st, 2010, respectively.

During his study in NUDT, he participated in several projects on image processing referring to objects detection and license plate recognition and so on from Oct.2008 to Dec.2010. As a result of his research work, he has two papers accepted by international conferences indexed by EI and ISTP or core Chinese academic journals. His previous and current research interests include: image processing, pattern recognition, computer simulation, and artificial intelligence. 